\title{
CLASS NUMBER FORMULAE IN THE FORM OF A PRODUCT OF DETERMINANTS IN FUNCTION FIELDS
}

\author{
JAEHYUN AHN, SOYOUNG CHOI and HWANYUP JUNG
}

(Received 23 October 2002; revised 16 October 2003)

\author{
Communicated by W. W. L. Chen
}

\begin{abstract}
In this paper, we generalize the Kučera's group-determinant formulae to obtain the real and relative class number formulae of any subfield of cyclotomic function fields with arbitrary conductor in the form of a product of determinants. From these formulae, we generalize the relative class number formula of Rosen and Bae-Kang and obtain analogous results of Tsumura and Hirabayashi for an intermediate field in the tower of cyclotomic function fields with prime power conductor.
\end{abstract}

2000 Mathematics subject classification: primary 11R60, 11 R58.

Keywords and phrases: cyclotomic function fields, class number.

\section{Introduction}

In the classical case, Tsumura [10] and Hirabayashi [4] gave relative class number formula for an intermediate field of the cyclotomic $\mathbb{Z}_{p}$-extension of an imaginary abelian number field in the form of a product of determinants. Recently Kučera [6] showed that the similar construction can be done for any extension of abelian fields using group determinant formulae.

In this paper, we generalize the group-determinant formulae of Kučera [6, Lemma 2] to obtain the real and relative class number formula of any subfield of cyclotomic function fields with arbitrary conductor in the form of a product of determinants (Theorem 3.1). As an application, we generalize the relative class number formula of Rosen [8] and Bae-Kang [2] to any subfield of cyclotomic function fields with arbitrary conductor (Theorem 3.2). We also give determinant formulae for the real and relative class number of an intermediate field in the tower of cyclotomic function fields with prime power conductor (Theorem 3.4 and Proposition 3.6).

This work was supported by the Korean Research Foundation Grant (KRF-2002-070-C00003).

(C) 2005 Australian Mathematical Society 1446-7887/05 $\$$ A2.00+0.00 


\section{Group-determinant formulae}

Let $G$ be a finite abelian group of order $n$ and let $L^{2}(G)$ denote the $n$-dimensional vector space of complex-valued functions on $G$. Let $\widehat{G}$ be the character group of $G$ with values in $\mathbb{C}$ and let $\chi_{0}$ be the trivial character of $G$. Then it is easy to see that $\widehat{G}$ is a basis for $L^{2}(G)$ over $\mathbb{C}$. For a subgroup $H$ of $G$, we define

$$
L^{2}(G)^{H}=\left\{f \in L^{2}(G): f(\sigma \tau)=f(\sigma) \text { for all } \sigma \in G \text { and } \tau \in H\right\}
$$

and $\widehat{G}^{H}=\{\chi \in \widehat{G}: \chi(\sigma)=1$ for all $\sigma \in H\}$. Then it is easy to see that $\widehat{G}^{H}=\widehat{G} \cap$ $L^{2}(G)^{H}$ and $\widehat{G}^{H}$ is naturally isomorphic to $\widehat{G / H}$ ([7, Lemmas 4.4-4.5]). Throughout the paper, we fix a family $\left\{\mathscr{R}_{G / H}\right\}_{H}$ of systems of representatives of $G / H$ for each subgroup $H$ of $G$ satisfying the following two conditions; (i) $1 \in \mathscr{R}_{G / H}$, (ii) if $H<H^{\prime}$, then $\mathscr{R}_{G / H} \supset \mathscr{R}_{G / H^{\prime}}$. For each subgroup $H$ of $G$, we define a function $r_{H}: G \rightarrow \mathscr{R}_{G / H}$ such that $r_{H}(\sigma) H=\sigma H$ for each $\sigma \in G$. For $f \in L^{2}(G)$, we define $s_{f}^{H} \in L^{2}(G)$ by $s_{f}^{H}(\sigma)=\sum_{\tau \in H} f(\sigma \tau)$ for each $\sigma \in G$. Clearly, $s_{f}^{H} \in L^{2}(G)^{H}$. Note that if $f \in L^{2}(G)^{H}$, then $s_{f}^{H}=|H| f$.

PROPOSITION 2.1. For $f \in L^{2}(G)$ and a subgroup $H$ of $G$, we have

$$
\prod_{\chi \in \widehat{G}^{H}} \sum_{\sigma \in G} \chi(\sigma) f(\sigma)=\operatorname{det}\left(s_{f}^{H}\left(\sigma \tau^{-1}\right)\right)_{\sigma, \tau},
$$

where $\sigma, \tau$ run through $\mathscr{R}_{G / H}$. Let $H^{\prime}$ be another subgroup of $G$ with $H^{\prime}>H$. Then we have

$$
\prod_{\chi \in \widehat{G}^{H} \backslash \widehat{G}^{H^{\prime}}} \sum_{\sigma \in G} \chi(\sigma) f(\sigma)=\operatorname{det}\left(s_{f}^{H}\left(\sigma \tau^{-1}\right)-s_{f}^{H}\left(\sigma r_{H^{\prime}}(\tau)^{-1}\right)\right)_{\sigma, \tau}
$$

where $\sigma, \tau$ run through $\mathscr{R}_{G / H} \backslash \mathscr{R}_{G / H^{\prime}}$

PRoof. Let $s(H)=\sum_{\sigma \in H} \sigma \in \mathbb{Z}[G]$ and $e_{H}=s(H) /|H|$ for any subgroup $H$ of $G$. Let $e_{\chi}=(1 /|G|) \sum_{\sigma \in G} \chi(\sigma) \sigma^{-1} \in \mathbb{C}[G]$ be the idempotent element associated to $\chi \in \widehat{G}$. Consider $\mathbb{C}[G]^{H}=\left\{x \in \mathbb{C}[G]: e_{H} x=x\right\}$, which is a $\mathbb{C}$-subspace of $\mathbb{C}[G]$. Both $X_{1}=\left\{\sigma s(H): \sigma \in \mathscr{R}_{G / H}\right\}$ and $X_{2}=\left\{e_{\chi}: \chi \in \widehat{G}^{H}\right\}$ are $\mathbb{C}$-bases of $\mathbb{C}[G]^{H}$. We consider the linear transformation $\Psi: \mathbb{C}[G]^{H} \rightarrow \mathbb{C}[G]^{H}$ defined as multiplication by $\theta=\sum_{\sigma \in G} f(\sigma) \sigma$. It is easy to see that matrices of $\Psi$ with respect to $X_{1}$ and $X_{2}$ are $\left(s_{f}^{H}\left(\sigma \tau^{-1}\right)\right)_{\sigma, \tau \in \mathscr{R}_{G / H}}$ and a diagonal matrix $\operatorname{diag}\left(\sum_{\sigma \in G} \chi(\sigma) f(\sigma)\right)_{\chi \in \widehat{G}^{H}}$, respectively. By comparing determinants of these two matrices, we get (1).

For (2), we consider the subspace $\mathbb{C}[G]_{H^{\prime}}^{H}=\left\{x \in \mathbb{C}[G]^{H}: e_{H^{\prime}} x=0\right\}$ of $\mathbb{C}[G]^{H}$. Both $Y_{1}=\left\{\left(\sigma-r_{H^{\prime}}(\sigma)\right) s(H): \sigma \in \mathscr{R}_{G / H} \backslash \mathscr{R}_{G / H^{\prime}}\right\}$ and $Y_{2}=\left\{e_{\chi}: \chi \in \widehat{G}^{H} \backslash \widehat{G}^{H^{\prime}}\right\}$ are $\mathbb{C}$-bases of $\mathbb{C}[G]_{H^{\prime}}^{H}$. We consider the restriction of $\Psi$ on $\mathbb{C}[G]_{H^{\prime}}^{H}$, which is also denoted 
by $\Psi$. Then the matrix of $\Psi$ with respect to $Y_{2}$ is $\operatorname{diag}\left(\sum_{\sigma \in G} \chi(\sigma) f(\sigma)\right)_{\chi \in \widehat{G}^{H} \backslash \widehat{G}^{H^{\prime}}}$. To compute the matrix of $\Psi$ with respect to $Y_{1}$, we need to consider $\theta\left(\tau_{1}-r_{H^{\prime}}\left(\tau_{1}\right)\right) s(H)$ for $\tau_{1} \in \mathscr{R}_{G / H} \backslash \mathscr{R}_{G / H^{\prime}}$. Then, we have

$$
\begin{aligned}
\theta\left(\tau_{1}\right. & \left.-r_{H^{\prime}}\left(\tau_{1}\right)\right) s(H) \\
= & \sum_{\sigma_{1} \in \mathscr{R}_{G / H}} \sum_{\sigma_{2} \in H} f\left(\sigma_{1} \sigma_{2}\right) \sigma_{1}\left(\tau_{1}-r_{H^{\prime}}\left(\tau_{1}\right)\right) s(H) \\
= & \sum_{\sigma_{1} \in \mathscr{Q}_{G / H}} \sum_{\sigma_{2} \in H} f\left(\sigma_{1} \sigma_{2}\right)\left(\left(\sigma_{1} \tau_{1}-r_{H^{\prime}}\left(\sigma_{1} \tau_{1}\right)\right)-\left(\sigma_{1} r_{H^{\prime}}\left(\tau_{1}\right)-r_{H^{\prime}}\left(\sigma_{1} \tau_{1}\right)\right)\right) s(H) \\
= & \sum_{\sigma_{1} \in \mathscr{R}_{C / H}} \sum_{\sigma_{2} \in H} f\left(\sigma_{1} \sigma_{2}\right)\left(\sigma_{1} \tau_{1}-r_{H^{\prime}}\left(\sigma_{1} \tau_{1}\right)\right) s(H) \\
& -\sum_{\sigma_{1} \in \mathscr{R}_{G / H}} \sum_{\sigma_{2} \in H} f\left(\sigma_{1} \sigma_{2}\right)\left(\sigma_{1} r_{H^{\prime}}\left(\tau_{1}\right)-r_{H^{\prime}}\left(\sigma_{1} r_{H^{\prime}}\left(\tau_{1}\right)\right)\right) s(H),
\end{aligned}
$$

where the last equality follows from the fact that $r_{H^{\prime}}\left(\sigma_{1} r_{H^{\prime}}\left(\tau_{1}\right)\right)=r_{H^{\prime}}\left(\sigma_{1} \tau_{1}\right)$. Replacing $\sigma_{1}$ by $r_{H}\left(\tau_{1}^{-1} \tau_{2}\right)$ and $r_{H}\left(r_{H^{\prime}}\left(\tau_{1}\right)^{-1} \tau_{2}\right)$ in the first and second summation of (3), respectively, we have that

$$
\begin{aligned}
\theta\left(\tau_{1}-r_{H^{\prime}}\left(\tau_{1}\right)\right) s(H) & \\
= & \sum_{\tau_{2} \in \mathscr{R}_{G / H} \backslash \mathscr{R}_{C_{/} H^{\prime}}}\left(\sum_{\sigma_{2} \in H} f\left(\tau_{1}^{-1} \tau_{2} \sigma_{2}\right)-\sum_{\sigma_{2} \in H} f\left(r_{H^{\prime}}\left(\tau_{1}^{-1}\right) \tau_{2} \sigma_{2}\right)\right)\left(\tau_{2}-r_{H^{\prime}}\left(\tau_{2}\right)\right) s(H),
\end{aligned}
$$

and so $\left(s_{f}^{H}\left(\sigma \tau^{-1}\right)-s_{f}^{H}\left(\sigma r_{H^{\prime}}(\tau)^{-1}\right)\right)_{\sigma, \tau \in \mathscr{R}_{G / H} \backslash \mathscr{R}_{G / H^{\prime}}}$ is the matrix of $\Psi$ with respect to $Y_{1}$. Thus we get (2).

COROLlary 2.2. For $f \in L^{2}(G)$ and a subgroup $H$ of $G$, we have

(i) $\prod_{\chi 0 \neq \chi \in \widehat{C}^{H}} \sum_{\sigma \in G} \chi(\sigma) f(\sigma)=\operatorname{det}\left(s_{f}^{H}\left(\sigma \tau^{-1}\right)-s_{f}^{H}(\sigma)\right)_{1 \neq \sigma, \tau \in \mathscr{R}_{G / H}}$.

(ii) $\prod_{\chi \in \widehat{G} \backslash \widehat{G}^{H}} \sum_{\sigma \in G} \chi(\sigma) f(\sigma)=\operatorname{det}\left(f\left(\sigma \tau^{-1}\right)-f\left(\sigma r_{H}(\tau)^{-1}\right)\right)_{\sigma, \tau \notin \mathscr{R}_{G / H}}$.

Let $H, H^{\prime}$ be any two subgroups of $G$ with $H<H^{\prime}$. Let $\left\{\psi_{1}, \ldots, \psi_{\left|H^{\prime}\right|}\right\}$ be a fixed system of representatives of $\widehat{G} / \widehat{G}^{H^{\prime}}$. Then $\left\{\psi_{1}, \ldots, \psi_{\left|H^{\prime}\right|}\right\} \cap \widehat{G}^{H}$ is a system of representatives of $\widehat{G}^{H} / \widehat{G}^{H^{\prime}}$. Note that $\widehat{G} / \widehat{G}^{H^{\prime}}$ (respectively $\widehat{G}^{H} / \widehat{G}^{H^{\prime}}$ ) is isomorphic to $\widehat{H^{\prime}}$ (respectively $\widehat{H^{\prime} / H}$ ) by restricting a character of $G$ to $H^{\prime}$.

We assume that $\left\{\psi_{1}, \ldots, \psi_{\left|H^{\prime}\right|}\right\} \cap \widehat{G}^{H}=\left\{\psi_{1}, \ldots, \psi_{\left|H^{\prime}\right| /|H|}\right\}$.

Corollary 2.3. Let $H, H^{\prime}$ and $\left\{\psi_{i}\right\}_{i}$ be as above. Then for any $f \in L^{2}(G)$, we have

(i) $\prod_{i=1}^{\left|H^{\prime}\right| /|H|} \operatorname{det}\left(s_{\psi_{i} f}^{H^{\prime}}\left(\sigma \tau^{-1}\right)\right)_{\sigma, \tau}=\left(\sum_{\sigma \in G} f(\sigma)\right) \operatorname{det}\left(s_{f}^{H}\left(\sigma \tau^{-1}\right)-s_{f}^{H}(\sigma)\right)_{\sigma, \tau}$, where $\sigma, \tau$ run through $\mathscr{R}_{G / H^{\prime}}$ in the left-hand side and run through $\mathscr{R}_{G / H} \backslash\{1\}$ in the righthand side. 
(ii) $\prod_{i=\left|H^{\prime}\right| /|H|+1}^{\left|H^{\prime}\right|} \operatorname{det}\left(s_{\psi_{i} f}^{H^{\prime}}\left(\sigma \tau^{-1}\right)\right)_{\sigma, \tau}=\operatorname{det}\left(f\left(\sigma \tau^{-1}\right)-f\left(\sigma r_{H}(\tau)^{-1}\right)\right)_{\sigma, \tau^{\prime}}$ where $\sigma, \tau$ run through $\mathscr{R}_{G / H^{\prime}}$ in the left-hand side and run through $G \backslash \mathscr{R}_{G / H}$.

PROOF. From Proposition 2.1 (i), we have

$$
\operatorname{det}\left(s_{\psi_{i} f}^{H^{\prime}}\left(\sigma \tau^{-1}\right)\right)_{\sigma, \tau \in \mathscr{R}_{G / H^{\prime}}}=\prod_{\chi \in \widehat{G}^{H^{\prime}}} \sum_{\sigma \in G} \chi(\sigma) \psi_{i}(\sigma) f(\sigma)=\prod_{\chi \in \psi_{i} \widehat{\widehat{G}}^{H^{\prime}}} \sum_{\sigma \in G} \chi(\sigma) f(\sigma) .
$$

Note that $\widehat{G}^{H}$ (respectively $\widehat{G} \backslash \widehat{G}^{H}$ ) is equal to the disjoint union of cosets $\psi_{i} \widehat{G}^{H^{\prime}}$ for $i=1, \ldots,\left|H^{\prime}\right| /|H|$ (respectively for $i=\left|H^{\prime}\right| /|H|+1, \ldots,\left|H^{\prime}\right|$ ). Thus we have

$$
\begin{aligned}
\prod_{i=1}^{\left|H^{\prime}\right| /|H|} \operatorname{det}\left(s_{\psi_{i} f}^{H^{\prime}}\left(\sigma \tau^{-1}\right)\right)_{\sigma, \tau \in \mathscr{R}_{G / H^{\prime}}} & =\prod_{\chi \in \widehat{G}^{H}} \sum_{\sigma \in G} \chi(\sigma) f(\sigma) \\
& =\left(\sum_{\sigma \in G} f(\sigma)\right) \prod_{\chi 0} \sum_{\chi \in \in \widehat{G}^{H}} \chi(\sigma) f(\sigma)
\end{aligned}
$$

and

$$
\prod_{i=\left|H^{\prime}\right| /|H|+1}^{\left|H^{\prime}\right|} \operatorname{det}\left(s_{\psi_{i} f}^{H^{\prime}}\left(\sigma \tau^{-1}\right)\right)_{\sigma, \tau \in \mathscr{R}_{G / H^{\prime}}}=\prod_{\chi \in \widehat{G} \backslash \widehat{G}^{H}} \sum_{\sigma \in G} \chi(\sigma) f(\sigma) .
$$

Thus the results follow from Corollary 2.2.

LEMMA 2.4. Let $J, H$ be two subgroups of $G$ with $J \cap H=\{1\}$. Then $\widehat{G}=\widehat{G}^{J} \widehat{G}^{H}$.

PROOF. Since $J \cap H=\{1\}$, we can choose $\mathscr{R}_{G / J}$ a system of representatives of $G / J$ containing $H$. Let $\psi_{J}=\psi \circ r_{J}$ for any $\psi \in \widehat{G}$. Then $\psi_{J} \in \widehat{G}^{J}$ and $\psi_{J}(\sigma)=\psi\left(r_{J}(\sigma)\right)=\psi(\sigma)$ for any $\sigma \in H$. Thus $\psi \psi_{J}^{-1} \in \widehat{G}^{H}$ and so $\widehat{G} \subseteq \widehat{G}^{J} \widehat{G}^{H}$, which proves the lemma.

Let $J, H$ be two subgroups of $G$ with $J \cap H=\{1\}$. By Lemma 2.4, we can choose $\left\{\psi_{1}, \ldots, \psi_{|H|}\right\}$ a system of representatives of $\widehat{G} / \widehat{G}^{H}$ such that $\psi_{i} \in \widehat{G}^{J}$ for all $i$. Note that $\widehat{H}$ consists of restrictions of $\psi_{i}$ to $H$. Taking $H^{\prime}=J H$ in Proposition 2.1, we have

$$
\begin{aligned}
\operatorname{det}\left(s_{\psi_{i} f}^{H}\left(\sigma \tau^{-1}\right)-s_{\psi_{i} f}^{H}\left(\sigma r_{J H}(\tau)^{-1}\right)\right)_{\sigma, \tau \in \mathscr{R}_{G / H} \backslash \mathscr{R}_{G / J H}} & =\prod_{\chi \in \widehat{\sigma}^{H} \backslash \hat{G}^{J H}} \sum_{\sigma \in G} \chi(\sigma) \psi_{i}(\sigma) f(\sigma)=\prod_{\chi \psi_{i}\left(\hat{G}^{H} \backslash \hat{G}^{J H}\right)} \sum_{\sigma \in G} \chi(\sigma) f(\sigma) .
\end{aligned}
$$

Since $\widehat{G} \backslash \widehat{G}^{J}$ is a disjoint union of $\psi_{i}\left(\widehat{G}^{H} \backslash \widehat{G}^{J H}\right)$ for $i=1, \ldots,|H|$, from Corollary 2.2 (ii), we have 
PROPOSITION 2.5. Let $J, H$ and $\left\{\psi_{i}\right\}_{i}$ be as above. Then for any $f \in L^{2}(G)$, we have

$$
\begin{gathered}
\prod_{i=1}^{|H|} \operatorname{det}\left(s_{\psi_{i} f}^{H}\left(\sigma \tau^{-1}\right)-s_{\psi_{i} f}^{H}\left(\sigma r_{J H}(\tau)^{-1}\right)\right)_{\sigma, \tau \in \mathscr{R}_{G / H} \backslash \mathscr{R}_{G / J H}} \\
=\operatorname{det}\left(f\left(\sigma \tau^{-1}\right)-f\left(\sigma r_{J}(\tau)^{-1}\right)\right)_{\sigma, \tau \notin \mathscr{R}_{G / J}} .
\end{gathered}
$$

\section{Class number formulae}

Let $\mathbb{A}=\mathbb{F}_{q}[T]$ be the polynomial ring over a finite field $\mathbb{F}_{q}$ with $q$ elements and let $\mathbb{F}_{q}(T)$ be the field of rational functions over $\mathbb{F}_{q}$. For each nonzero $N \in \mathbb{A}$, one uses the Carlitz module to construct a field extension $K_{N}$ over $\mathbb{F}_{q}(T)$, called the $N$-th cyclotomic function field and its maximal real subfield $K_{N}^{+}$. For more details on Carlitz module and cyclotomic theory of function field, we refer to Hayes' paper [3] and Rosen's book [9, Chapter 12]. It is known [9, Theorems 12.8, 12.14] that $G_{N}=\operatorname{Gal}\left(K_{N} / \mathbb{F}_{q}(T)\right)$ is isomorphic to $(\mathbb{A} / N \mathbb{A})^{*}$ and $\operatorname{Gal}\left(K_{N} / K_{N}^{+}\right)$is isomorphic to $\mathbb{F}_{q}^{*}$. For any $A \in \mathbb{A}$ which is relatively prime to $N$, we denote by $\sigma_{A}$ the element of $\operatorname{Gal}\left(K_{N} / \mathbb{F}_{q}(T)\right)$ corresponding to $A \bmod N \mathrm{~A}$. Any character $\chi \in \widehat{G}_{N}$ may be viewed as a primitive Dirichlet character, and so the conductor $F_{\chi}$ of $\chi$ which is a monic polynomial is defined. For $\chi \in \widehat{G}_{N}$ and an irreducible polynomial $P \in \mathbb{A}$, we define $\chi(P)=\chi\left(\left(P, K_{F_{\chi}} / \mathbb{F}_{q}(T)\right)\right)$ if $P \nmid F_{\chi}$ and $\chi(P)=0$ otherwise. Here $\left(P, K_{F_{x}} / F_{q}(T)\right)$ denotes the Artin automorphism of $P$ in $K_{F_{x}}$. We extend $\chi$ to a function from $\mathbb{A}$ to $\mathbb{C}$ using irreducible factorization of polynomials. For a non zero $N \in \mathbb{A}$, let $\Phi(N)$ be the order of $(\mathbb{A} / N \mathbb{A})^{*}$, and let $\mathbb{M}_{N}$ be the set of polynomials of degree less than $\operatorname{deg} N$ and relatively prime to $N$. Let $\mathbb{M}_{N}^{+}$be the subset of $\mathbb{M}_{N}$ consisting of monic ones and $\mathbb{M}_{N}^{-}=\mathbb{M}_{N} \backslash \mathbb{M}_{N}^{+}$.

Let $k$ be any abelian extension of $\mathbb{F}_{q}(T)$ with conductor $N$, that is, $K_{N}$ is the smallest cyclotomic function field containing $k$. Let $k^{+}=k \cap K_{N}^{+}$be the maximal real subfield of $k$. Let $G=\operatorname{Gal}\left(k / \mathbb{F}_{q}(T)\right), G^{+}=\operatorname{Gal}\left(k^{+} / \mathbb{F}_{q}(T)\right)$ and $J=\operatorname{Gal}\left(k / k^{+}\right)$. We denote $\widehat{G}^{J}$ by $\widehat{G}^{+}$and $\widehat{G} \backslash \widehat{G}^{J}$ by $\widehat{G}^{-}$. Let $h(k)$ and $h\left(k^{+}\right)$be the divisor class number of $k$ and $k^{+}$, respectively. It is known that $h(k)$ is divisible by $h\left(k^{+}\right)$. Let $h^{-}(k)=h(k) / h\left(k^{+}\right)$, called the relative divisor class number of $k$. For $A \in \mathbb{A}$, relatively prime to $N$, let $\left.\sigma_{A}\right|_{k}$ be the restriction of $\sigma_{A}$ to $k$. By ' $F \mid N$ ' we mean that $F$ is a monic polynomial dividing $N$. Assume that we are given $\left\{a_{F, \sigma} \in \mathbb{Q}: 1 \neq\right.$ $F \mid N, \sigma \in G\}$. We define two functions $t_{1}, t_{2} \in L^{2}(G)$ as follows; for any $\sigma \in G$,

$$
\begin{aligned}
& t_{1}(\sigma)=\sum_{F \mid N, F \neq 1} \sum_{A \in \mathbb{M}_{N}} a_{F,\left.\sigma_{A}\right|_{k} \sigma^{-1}} \varphi(A / F), \\
& t_{2}(\sigma)=\sum_{F \mid N, F \neq 1} \sum_{A \in \mathbb{M}_{N}} a_{F,\left.\sigma_{A}\right|_{k} \sigma^{-1}} Z_{F}(0, A),
\end{aligned}
$$


where $\varphi(A / F)=(q-1)(\operatorname{deg} F-1-\operatorname{deg} \bar{A})-1$ with $\bar{A} \equiv A \bmod F, \operatorname{deg} \bar{A}<\operatorname{deg} F$ and $Z_{F}(s, A)$ is the partial zeta function associated to the class of $A$ in $C l(\mathbb{A})$. Let $r=\left[k^{+}: \mathbb{F}_{q}(T)\right]-1$. Define

$$
c_{\chi}=\sum_{F \mid N, F \neq 1} b_{F, \chi} \prod_{P \mid F}(1-\bar{\chi}(P)) \quad \text { with } \quad b_{F, \chi}=(\Phi(N) / \Phi(F)) \sum_{\sigma \in G} a_{F, \sigma} \chi(\sigma) .
$$

Then we have from $[1$, Theorems $3.1,3.6]$ that

$$
\begin{aligned}
\operatorname{det}\left(t_{1}\left(\sigma \tau^{-1}\right)-t_{1}(\sigma)\right)_{1 \neq \sigma, \tau \in \mathscr{R}_{G / J}} & = \pm \frac{(q-1)^{2 r}}{|J|^{r}} h\left(k^{+}\right) \prod_{\chi 0 \neq \chi \in \widehat{G}^{+}} c_{\chi}, \\
\operatorname{det}\left(t_{2}\left(\sigma \tau^{-1}\right)-t_{2}\left(\sigma r_{J}(\tau)^{-1}\right)\right)_{\sigma, \tau \notin \mathscr{R}_{C_{I} /}} & = \pm h^{-}(k) \prod_{x \in \widehat{G}^{-}} c_{X} .
\end{aligned}
$$

Let $k_{0}$ be a subfield of $k$ with $G_{0}=\operatorname{Gal}\left(k_{0} / \mathbb{F}_{q}(T)\right)$ and $G_{0}^{+}=\operatorname{Gal}\left(k_{0}^{+} / \mathbb{F}_{q}(T)\right)$. Let $H=\mathrm{Gal}\left(k / k_{0}\right)$. Choose $\left\{\psi_{i}: 1 \leq i \leq|J H|\right\}$ a system of representatives of $\widehat{G} / \widehat{G}^{J H}$ such that $\psi_{i} \in \widehat{G}^{+}$for $1 \leq i \leq|J H| /|J|$ and $\psi_{i} \in \widehat{G}^{-}$otherwise. Since $t_{1} \in L^{2}(G)^{J}$, we have $s_{\chi t_{1}}^{J}=|J| \chi t_{1} \in L^{2}(G)^{J}$ for any $\chi \in \widehat{G}^{+}$. By combining (4) and (5) with Corollary 2.3, we have the following.

THEOREM 3.1. Let $k_{0}$ be a subfield of $k, G=\operatorname{Gal}\left(k / \mathbb{F}_{q}(T)\right), G_{0}=\operatorname{Gal}\left(k_{0} / \mathbb{F}_{q}(T)\right)$ and $H=\operatorname{Gal}\left(k / k_{0}\right)$. With $\left\{\psi_{i}: 1 \leq i \leq|J H|\right\}$ a system of representatives of $\widehat{G} / \widehat{G}^{J H}$ as above, we have

(i) $\prod_{i=1}^{|J H| /|J|} \operatorname{det}\left(s_{\psi_{i} f_{1}}^{J H}\left(\sigma \tau^{-1}\right)\right)_{\sigma, \tau}= \pm(q-1)^{2 r} h\left(k^{+}\right)\left(\sum_{\sigma \in G} t_{1}(\sigma)\right) \prod_{\chi 0 \neq x \in \widehat{G}^{+}} c_{\chi}$,

(ii) $\prod_{i=|J H| /|J|+1}^{|J H|} \operatorname{det}\left(s_{\psi_{i} t_{2}}^{J H}\left(\sigma \tau^{-1}\right)\right)_{\sigma, \tau}= \pm h^{-}(k) \prod_{\chi \in \widehat{G}^{-}} c_{\chi}$,

where $\sigma, \tau$ run through $\mathscr{R}_{G / J H}$.

REMARK. From Corollary 2.3, (4) and (5), we see that $t_{1}$ (respectively $t_{2}$ ) in Theorem 3.1 can be replaced by any function $f \in L^{2}(G)$ of the form $f=t_{1}+c$ (respectively $f=t_{2}+c$ ), where $c \in \mathbb{C}$ is a constant.

3.1. Relative class number In this subsection we give a determinant formula for $h^{-}(k)$, which is a generalization of Rosen [8, Theorem 1] and Bae-Kang [2, Theorem 2]. We apply Theorem 3.1 (ii) with $k=k_{0}$. Recall that $k$ is an abelian extension of $\mathbb{F}_{q}(T)$ with conductor $N$. For any $A \in \mathbb{A}$, relatively prime to $N$, let $\bar{A} \in \mathbb{M}_{N}$ be the unique element such that $A \equiv \bar{A} \bmod N$ and $A^{\prime} \in \mathbb{M}_{N}$ be the unique element such that $A A^{\prime} \equiv 1 \bmod N$. We also let $\operatorname{sgn}_{N}(A)$ be the leading coefficient of $\bar{A}$ and $\operatorname{deg}_{N}(A)$ be the degree of $\bar{A}$. We define $\langle A\rangle_{N}=1$ if $\operatorname{sgn}_{N}(A)=1$ and 0 otherwise. From [5, Lemma 3.1], we know that $Z_{N}(0, A)=\langle A\rangle_{N}-1 /(q-1)$. Take $a_{F, \sigma}=1$ if $F=N, \sigma=1$ and 0 otherwise. Then $t_{2}(\sigma)=\sum_{A \in \mathbb{M}_{N},\left.\sigma_{A}\right|_{k}=\sigma} Z_{N}(0, A)$, 
and $b_{F, X}=1$. We define $f(\sigma)=\sum_{A \in M_{N},\left.\sigma_{A}\right|_{k}=\sigma}\langle A\rangle_{N}$ for any $\sigma \in G$. Then

$$
f(\sigma)=t_{2}(\sigma)-\left[K_{N}: k\right] /(q-1)
$$

for all $\sigma \in G$. Let $\left\{\psi_{i}: 1 \leq i \leq|J|\right\}$ be any set of representatives of $\widehat{G} / \widehat{G}^{+}$such that $\psi_{1} \in \widehat{G}^{+}$and $\psi_{i} \in \widehat{G}^{-}$for $2 \leq i \leq|J|$. Then by Theorem 3.1 (ii) and Remark 3, we have

$$
\prod_{i=2}^{|J|} \operatorname{det}\left(s_{\psi_{i} f}^{J}\left(\sigma \tau^{-1}\right)\right)_{\sigma, \tau \in \mathscr{R}_{C / J}}= \pm h^{-}(k) Q_{k}^{(-)},
$$

where $Q_{k}^{(-)}=\prod_{\chi \in \hat{G}^{-}} \prod_{P \mid N}(1-\bar{\chi}(P))$. Now we consider $s_{\psi_{i} f}^{J}(\sigma)$ for $\sigma \in G$. From its definition, we have

$$
\begin{aligned}
s_{\psi_{i} f}^{J}(\sigma) & =\sum_{\substack{\left.\tau \in G \\
\tau\right|_{k^{+}}=\left.\sigma\right|_{k^{+}}}} \psi_{i}(\tau) f(\tau)=\sum_{\substack{\left.\tau \in G \\
\tau\right|_{k^{+}}=\left.\sigma\right|_{k^{+}}}} \psi_{i}(\tau) \sum_{\substack{\left.A \in \mathbb{M}_{N} \\
\sigma_{A}\right|_{k}=\tau}}\langle A\rangle_{N} \\
& =\sum_{\substack{A \in \mathbb{M}_{N} \\
\sigma_{A}\left|k^{+}=\sigma\right|_{k^{+}}}} \psi_{i}\left(\sigma_{A}\right)\langle A\rangle_{N}=\sum_{\substack{\left.A \in \mathbb{M}_{N}^{+} \\
\sigma_{A}\right|_{k^{+}}=\left.\sigma\right|_{k^{+}}}} \psi_{i}\left(\sigma_{A}\right) .
\end{aligned}
$$

Here we also view $\psi_{i}$ as a character of $G_{N}$ under the canonical inclusion map $\widehat{G} \hookrightarrow$ $\widehat{G}_{N}$. Choose a subset $\mathscr{R}$ of $\mathbb{M}_{N}^{+}$such that $G^{+}=\left\{\left.\sigma_{A}\right|_{k^{+}}: A \in \mathscr{R}\right\}$ with $|\mathscr{R}|=\left|G^{+}\right|$and a subset $\Delta$ of $\mathbb{M}_{N}$ such that $\operatorname{Gal}\left(K_{N} / k\right)=\left\{\sigma_{A}: A \in \Delta\right\}$ with $|\Delta|=\left|\mathrm{Gal}\left(K_{N} / k\right)\right|$. Let $\Delta^{+}=\Delta \cap \mathbb{M}_{N}^{+}$. Clearly $\left\{\sigma_{A}: A \in \mathbb{F}_{q}^{*} \Delta\right\}=\left\{\sigma_{A}: A \in \mathbb{F}_{q}^{*} \Delta^{+}\right\}=\operatorname{Gal}\left(K_{N} / k^{+}\right)$and so, for any $A \in \mathbb{A}$, we have $\left\{B \in \mathbb{M}_{N}:\left.\sigma_{B}\right|_{k^{+}}=\left.\sigma_{A}\right|_{k^{+}}\right\}=\left\{\overline{A(\alpha D)}: \alpha \in \mathbb{F}_{q}^{*}, D \in \Delta^{+}\right\}$. Thus $\left\{B \in \mathbb{M}_{N}^{+}:\left.\sigma_{B}\right|_{k^{+}}=\left.\sigma_{A}\right|_{k^{+}}\right\}=\left\{\overline{A D} \operatorname{sgn}_{N}(A D)^{-1}: D \in \Delta^{+}\right\}$. From this, for $\sigma=\left.\sigma_{A}\right|_{k}, \tau=\left.\sigma_{B}\right|_{k} \in G$, we have

$$
\begin{aligned}
s_{\psi_{i} f}^{J}\left(\sigma \tau^{-1}\right) & =\sum_{D \in \Delta^{+}} \psi_{i}\left(A B^{\prime} D \operatorname{sgn}_{N}\left(A B^{\prime} D\right)^{-1}\right) \\
& =\psi_{i}(A) \overline{\psi_{i}}(B) \sum_{D \in \Delta^{+}} \overline{\psi_{i}}\left(\operatorname{sgn}_{N}\left(A B^{\prime} D\right)\right),
\end{aligned}
$$

because $\left\{\sigma_{A}: A \in \Delta\right\}=\operatorname{Gal}\left(K_{N} / k\right)$. Here we view $\psi_{i}$ as a primitive Dirichlet character. Thus

$$
\operatorname{det}\left(s_{\psi_{i} f}^{J}\left(\sigma \tau^{-1}\right)\right)_{\sigma, \tau \in \mathscr{R}_{G / J}}=\operatorname{det}\left(\sum_{D \in \Delta^{+}} \overline{\psi_{i}}\left(\operatorname{sgn}_{N}\left(A B^{\prime} D\right)\right)\right)_{A, B \in \mathscr{R}} .
$$

It is easy to see that restrictions of $\psi_{2}, \ldots, \psi_{|J|}$ to $\mathbb{F}_{q}^{*}$ are all non-trivial characters of $J$. Thus we have the following theorem. 
THEOREM 3.2. Let $k$ be a finite abelian extension of $\mathbb{F}_{q}(T)$ with conductor $N$. Let $\Delta^{+}, \mathscr{R}$ be subsets of $\mathbb{M}_{N}^{+}$as above. Then we have

$$
\prod_{\lambda \neq \lambda_{0}} \operatorname{det}\left(\sum_{D \in \Delta^{+}} \lambda\left(\operatorname{sgn}_{N}\left(A B^{\prime} D\right)\right)\right)_{A, B \in \mathscr{R}}= \pm h^{-}(k) Q_{k}^{(-)}
$$

where $\lambda$ runs over all non-trivial characters of $J$.

REMARK. Let $P \in A$ be a monic irreducible polynomial. When $k=K_{P^{n}}$, we have $Q_{k}^{(-)}=1\left(\left[1\right.\right.$, Lemma 3.7]), $\mathscr{R}=\mathbb{M}_{P^{n}}$ and $\Delta^{+}=\{1\}$ and so Theorem 3.2 gives the determinant class number formula of Rosen and Bae-Kang for $h^{-}\left(K_{P^{n}}\right)$. We also note that $\widehat{J}=\left\langle\lambda^{(q-1) /|J|}\right\rangle$ for any generator $\lambda$ of $\widehat{\mathbb{F}}_{q}^{*}$.

Since $Q_{k}^{(-)}$is zero in some cases (see [1, Lemma 3.7]), it will be interesting to give a non-vanishing version of the above determinant class number formula. This can be done as in [1, Section 3]. Let $N=\prod_{i=1}^{s} P_{i}^{r_{i}}$ be the factorization of $N$ into monic irreducible polynomials. Let $S=\{1, \ldots, s\}$ and $N_{l}=\prod_{i \in l} P_{i}^{r_{i}}$ for $I \subseteq S$. Define $f \in L^{2}(G)$ by $f(\sigma)=\sum_{I \subsetneq S}(-1)^{|I|} \Phi\left(N_{l}\right)^{-1} \sum_{A \in M_{N},\left.\sigma_{A}\right|_{k}=\sigma}\langle A\rangle_{N / N_{l}}$ for any $\sigma \in G$. With this choice of $f$, using [1, Proposition 3.11] and Theorem 3.1, we have

$$
\prod_{i=2}^{|J|} \operatorname{det}\left(s_{\psi_{i} f}^{J}\left(\sigma \tau^{-1}\right)\right)_{\sigma, \tau \in \mathscr{R}_{G / J}}= \pm h^{-}(k)
$$

By choice of $\mathscr{R},\left\{\left.\sigma_{A}\right|_{k}: A \in \mathscr{R}\right\}$ is a system of representatives of $G / J$. It is easy to see that

$$
s_{\chi f}^{J}\left(\sigma \tau^{-1}\right)=\chi\left(\sigma_{A}\right) \bar{\chi}\left(\sigma_{B}\right) \sum_{I \subsetneq S}(-1)^{|l|} \Phi\left(N_{I}\right)^{-1} \sum_{D \in \Delta^{+}} \bar{\chi}\left(\operatorname{sgn}_{N / N_{I}}\left(A B^{\prime} D\right)\right),
$$

for any $\chi \in \widehat{G}^{-}$and $\sigma=\left.\sigma_{A}\right|_{k}, \tau=\left.\sigma_{B}\right|_{k}$ with $A, B \in \mathscr{R}$. Thus we have

PROPOSITION 3.3.

$$
\prod_{\lambda \neq \lambda_{0}} \operatorname{det}\left(\sum_{I \subsetneq S}(-1)^{|I|} \Phi\left(N_{I}\right)^{-1} \sum_{D \in \Delta^{+}} \bar{\chi}\left(\operatorname{sgn}_{N / N_{I}}\left(A B^{\prime} D\right)\right)\right)_{A, B \in \mathscr{R}}= \pm h^{-}(k)
$$

where $\lambda$ runs over all non-trivial characters of $J$. 
3.2. Tower of cyclotomic function fields In this subsection we give real and relative class number formulae for an intermediate field in the tower of cyclotomic function fields with prime power conductor $K_{P} \subset K_{P^{2}} \subset \cdots \subset K_{P^{n+1}} \subset \cdots$ as a product of determinants of matrices of the fixed degree $\Phi(P) /(q-1)$. We apply Theorem 3.1 with $k=K_{P^{n+1}}$ and $k_{0}=K_{P}$. In this case $\left.G=\operatorname{Gal}\left(K_{P^{n+1}}\right) / \mathbb{F}_{q}(T)\right), H=\operatorname{Gal}\left(K_{P^{n+1}} / K_{P}\right)$ and $J=\left\{\sigma_{\alpha}: \alpha \in \mathbb{F}_{q}^{*}\right\}$. Let $d=\operatorname{deg}(P)$. For convenience we assume that $\operatorname{deg}(0)=-\infty$. Define

$$
\mathscr{M}_{P^{n+1}}^{+}=\prod_{\chi \in \widehat{H}}\left(\sum_{\operatorname{deg} D<n d} \chi(1+D P) \operatorname{deg}_{P^{n+1}}\left(A B^{\prime}(1+D P)\right)\right)_{A, B \in \mathbb{M}_{P}^{+}}
$$

and

$$
\mathscr{M}_{P^{n+1}}^{-}=\prod_{\lambda \neq \lambda_{0}} \prod_{\chi} \in\left(\sum_{\operatorname{deg} D<n d} \chi(1+D P) \lambda\left(\operatorname{sgn}_{P^{n+1}}\left(A B^{\prime}(1+D P)\right)\right)\right)_{A, B \in \mathbb{M}_{P}^{+}},
$$

where $\lambda$ runs over all non-trivial characters of $\mathbb{F}_{q}^{*}$.

THEOREM 3.4. For a monic irreducible polynomial $P$ of $\mathbb{A}$, let $N=p^{n+1}$ with $n \geq 0$ integer. Let $\mathscr{M}_{N}^{+}$and $\mathscr{M}_{N}^{-}$be defined as above. Then we have

(i) $\operatorname{det} \mathscr{M}_{N}^{+}= \pm h\left(K_{N}^{+}\right)\left(\sum_{A \in \mathbb{M}_{N}^{+}} \operatorname{deg} A\right)$;

(ii) $\operatorname{det} \mathscr{M}_{N}^{-}= \pm h^{-}\left(K_{N}\right)$.

Proof. Note that $H=\left\{\sigma_{1+D P} \in \operatorname{Gal}\left(K_{N} / \mathbb{F}_{q}(T)\right): D \in \mathbb{A}\right.$ with $\left.\operatorname{deg} D<\operatorname{deg} P^{n}\right\}$ and $\left\{\sigma_{A}: A \in \mathbb{M}_{P}^{+}\right\}$forms a system of representatives of $G / J H$. Since $J \cap H=$ $\{1\}$, from Lemma 2.4 , we can choose $\left\{\lambda_{0}, \ldots, \lambda_{|J|-1}\right\}$ a system of representatives of $\widehat{G} / \widehat{G}^{J}$ with $\lambda_{i} \in \widehat{G}^{H}$ and $\left\{\chi_{1}, \ldots, \chi_{|H|}\right\}$ a system of representatives of $\widehat{G} / \widehat{G}^{H}$ with $\chi_{j} \in \widehat{G}^{J}$. It is easy to see that $\left\{\left.\lambda_{i}\right|_{J}\right\}_{i}=\widehat{J},\left\{\left.\chi_{j}\right|_{H}\right\}_{j}=\widehat{H}$ and $\left\{\lambda_{i} \chi_{j}\right\}_{i, j}$ is a system of representatives of $\widehat{G} / \widehat{G}^{J H}$. We write $\left\{\psi_{1}, \ldots, \psi_{|J H|}\right\}=\left\{\lambda_{i} \chi_{j}: 0 \leq i \leq\right.$ $|J|-1,1 \leq j \leq|H|\}$. We may assume that $\lambda_{0}$ is the trivial character of $G$ and so $\left\{\psi_{1}, \ldots, \psi_{|H|}\right\}=\left\{\chi_{1}, \ldots, \chi_{|H|}\right\} \subset \widehat{G}^{+}$.

Take $a_{F, \sigma}=1$ if $F=N, \sigma=1$ and 0 otherwise. Then we have $t_{1}\left(\sigma_{A}\right)=\varphi(A / N)$ and $t_{2}\left(\sigma_{A}\right)=Z_{N}(0, A)$ for any $A \in \mathbb{M}_{N}$. Thus for $A, B \in \mathbb{M}_{N}$, we have

$$
t_{1}\left(\sigma_{A} \sigma_{B}\right)-t_{1}\left(\sigma_{A}\right)=-(q-1)\left(\operatorname{deg}_{N}(A B)-\operatorname{deg}_{N}(A)\right) .
$$

We note that $\prod_{\chi 0 \neq x \in \widehat{G}^{+}} c_{\chi}=\prod_{x \in \widehat{G}^{-}} c_{\chi}=1$ (see [1, Lemmas 3.2, 3.7]). Define $f\left(\sigma_{A}\right)=\operatorname{deg}_{N}(A)$ for any $A \in \mathbb{M}_{N}$. Then from Theorem 3.1 (i) and (4), we have

$$
\prod_{i=1}^{|H|} \operatorname{det}\left(s_{\psi ;}^{J H}\left(\sigma_{A} \sigma_{B}^{-1}\right)\right)_{A, B \in \mathbb{M}_{P}^{+}}= \pm(q-1)^{r} h\left(K_{N}^{+}\right) \sum_{A \in \mathbb{M}_{N}} \operatorname{deg}_{N}(A),
$$


where $r=\left[K_{N}^{+}: \mathbb{F}_{q}(T)\right]-1$. For $1 \leq i \leq|H|$ and $A, B \in \mathbb{M}_{p}^{+}$, we have

$$
\begin{aligned}
s_{\psi_{i} f}^{J H}\left(\sigma_{A} \sigma_{B}^{-1}\right) & =\sum_{\sigma \in J H} \psi_{i}\left(\sigma_{A} \sigma_{B}^{-1} \sigma\right) f\left(\sigma_{A} \sigma_{B}^{-1} \sigma\right) \\
& =(q-1) \sum_{\operatorname{deg} D<n d} \psi_{i}\left(\sigma_{A} \sigma_{B}^{-1} \sigma_{1+D P}\right) \operatorname{deg}_{N}\left(A B^{\prime}(1+D P)\right) \\
& =(q-1) \chi_{i}\left(\sigma_{A}\right) \bar{\chi}_{i}\left(\sigma_{B}\right) \sum_{\operatorname{deg} D<n d} \chi_{i}\left(\sigma_{1+D P}\right) \operatorname{deg}_{N}\left(A B^{\prime}(1+D P)\right),
\end{aligned}
$$

because $\left\{\psi_{1}, \ldots, \psi_{|H|}\right\}=\left\{\chi_{1}, \ldots, \chi_{|H|}\right\} \subset \widehat{G}^{+}$and $f \in L^{2}(G)^{J}$. Since

$$
\sum_{A \in \mathbb{M}_{N}} \operatorname{deg}_{N}(A)=(q-1) \sum_{A \in \mathbb{M}_{N}^{+}} \operatorname{deg}_{N}(A)
$$

and $r=|H|\left|\mathbb{M}_{P}^{+}\right|-1$, (i) follows from (7).

For (ii), we define $f\left(\sigma_{A}\right)=\langle A\rangle_{N}$ for any $A \in \mathbb{M}_{N}$. Then from Theorem 3.1 (ii) and Remark 3, we have

$$
\prod_{i=|H|+1}^{|J H|} \operatorname{det}\left(s_{\psi_{i} f}^{J H}\left(\sigma_{A} \sigma_{B}^{-1}\right)\right)_{A, B \in \mathbb{M}_{P}^{+}}= \pm h^{-}\left(K_{N}\right) .
$$

For $|H|+1 \leq i \leq|J H|$ and $A, B \in \mathbb{M}_{P}^{+}$, we have

$$
\begin{aligned}
s_{\psi_{i} f}^{J H}\left(\sigma_{A} \sigma_{B}^{-1}\right) & =\sum_{\sigma \in J H} \psi_{i}\left(\sigma_{A} \sigma_{B}^{-1} \sigma\right) f\left(\sigma_{A} \sigma_{B}^{-1} \sigma\right) \\
& =\sum_{\alpha \in \mathbb{F}_{q}^{*} \operatorname{deg} D<n d} \sum_{i} \psi_{i}\left(\sigma_{A} \sigma_{B}^{-1} \sigma_{1+D P} \sigma_{\alpha}\right)\left\langle A B^{\prime}(1+D P) \alpha\right\rangle_{N} \\
& =\psi_{i}\left(\sigma_{A}\right) \bar{\psi}_{i}\left(\sigma_{B}\right) \sum_{\operatorname{deg} D<n d} \psi_{i}\left(\sigma_{1+D P}\right) \bar{\psi}_{i}\left(\operatorname{sgn}_{N}\left(A B^{\prime}(1+D P)\right)\right)
\end{aligned}
$$

Since $\left\{\psi_{i}:|H|+1 \leq i \leq|J H|\right\}=\left\{\lambda_{i} \chi_{j}: 1 \leq i \leq|J|-1,1 \leq j \leq|H|\right\}$, (ii) follows from (8).

For any integer $m \geq 1$ and any ring $R$, let $\operatorname{Mat}(m, R)$ denote the ring of $m \times m$ matrices with entries in $R$. As [10, Lemma 2.6], we have

LEMMA 3.5. For any integer $n \geq 0$ and monic irreducible polynomial $P$, we have that $\mathscr{M}_{P^{n+1}}^{+} \in \operatorname{Mat}(\Phi(P) /(q-1), \mathbb{Z})$ and $\mathscr{M}_{P^{n+1}}^{-} \in \operatorname{Mat}\left(\Phi(P) /(q-1), \mathbb{Z}\left[\zeta_{q-1}\right]\right)$.

Proof. Let $d=\operatorname{deg} P, N=P^{n+1}$ and $H=\operatorname{Gal}\left(K_{N} / K_{P}\right)$ as before. For any $C=\left(c_{i j}\right) \in \operatorname{Mat}\left(\Phi(P) /(q-1), \mathbb{Q}\left(\zeta_{q^{\text {nd }}}\right)\right)$ and $\sigma \in \operatorname{Gal}\left(\mathbb{Q}\left(\zeta_{q^{\text {nd }}}\right) / \mathbb{Q}\right)$, we let $C^{\sigma}=\left(c_{i j}^{\sigma}\right)$. 
Write

$$
\mathscr{M}_{N, \chi}^{+}=\left(\sum_{\operatorname{deg} D<n d} \chi(1+D P) \operatorname{deg}_{N}\left(A B^{\prime}(1+D P)\right)\right)_{A, B \in \mathbb{M}_{P}^{+}}
$$

so that $\mathscr{M}_{N}^{+}=\prod_{\chi} \mathscr{M}_{N_{\perp} \chi^{+}}^{+}$Since $|H|=q^{\text {nd }}$, for any $\sigma \in \operatorname{Gal}\left(\mathbb{Q}\left(\zeta_{q^{\text {nd }}}\right) / \mathbb{Q}\right)$, we have $\left\{\chi^{\sigma}: \chi \in \widehat{H}\right\}=\overparen{H}$. Thus for any $\sigma \in \operatorname{Gal}\left(\mathbb{Q}\left(\zeta_{q^{n d}}\right) / \mathbb{Q}\right)$, we have $\left(\mathscr{M}_{N}^{+}\right)^{\sigma}=$ $\prod_{\chi} \mathscr{M}_{N, \chi^{\alpha}}^{+}=\mathscr{M}_{N}^{+}$. Therefore $\mathscr{M}_{N}^{+} \in \operatorname{Mat}(\Phi(P) /(q-1), \mathbb{Z})$.

By considering the extension of $\sigma$ to $\operatorname{Gal}\left(\mathbb{Q}\left(\zeta_{q^{\text {nd }}(q-1)}\right) / \mathbb{Q}\left(\zeta_{q-1}\right)\right)$, we show that $\mathscr{M}_{P^{n+1}}^{-} \in \operatorname{Mat}\left(\Phi(P) /(q-1), \mathbb{Z}\left[\zeta_{q-1}\right]\right)$.

Let $f\left(\sigma_{A}\right)=\langle A\rangle_{P^{n+1}}$ for any $A \in \mathbb{M}_{P^{n+1}}$. Let $\left\{\psi_{1}, \ldots, \psi_{|H|}\right\}$ be a system of representatives of $\widehat{G} / \widehat{G}^{H}$ such that $\psi_{i} \in \widehat{G}^{+}$for all $i$. For any $A, B \in \mathbb{M}_{P}$, we have

$$
\begin{aligned}
s_{\psi_{i} f}^{H}\left(\sigma_{A} \sigma_{B}^{-1}\right) & =\sum_{\operatorname{deg} D<n d} \psi_{i}\left(\sigma_{A} \sigma_{B}^{-1} \sigma_{1+D P}\right) f\left(\sigma_{A} \sigma_{B}^{-1} \sigma_{1+D P}\right) \\
& =\psi_{i}\left(\sigma_{A}\right) \bar{\psi}_{i}\left(\sigma_{B}\right) \sum_{\operatorname{deg} D<n d} \psi_{i}\left(\sigma_{1+D P}\right)\left\langle A B^{\prime}(1+D P)\right\rangle_{P^{n+1}}
\end{aligned}
$$

Define $\mathscr{D}_{p^{n+1}}^{-}=\prod_{\chi \in \hat{H}}\left(\sum_{\operatorname{deg} D<n d} \chi(1+D P)\left\langle A B^{\prime}(1+D P)\right\rangle_{P^{n+1}}\right)_{A, B \in \mathbb{M}_{p}^{-}}$. By similar argument in the proof of Lemma 3.5, we see that $\mathscr{D}_{p^{n+1}}^{-} \in \operatorname{Mat}((q-2) /(q-1) \Phi(P), \mathbb{Z})$. Note that $\mathbb{M}_{P}^{-}$corresponds to $\mathscr{R}_{G / H} \backslash \mathscr{R}_{G / J H}$ bijectively under the map $A \mapsto \sigma_{A}$. Thus from Proposition 2.5, we have the following.

PROPOSITION 3.6. Let $P$ be a monic irreducible polynomial and $n \geq 0$ integer. Then we have $\operatorname{det}\left(\mathscr{D}_{P^{n+1}}^{-}\right)= \pm h^{-}\left(K_{P^{n+1}}\right)$ with $\mathscr{D}_{P^{n+1}}^{-} \in \operatorname{Mat}((q-2) /(q-1) \Phi(P), \mathbb{Z})$.

\section{Acknowledgement}

The authors would like to express their sincere gratitude to the referee for his careful reading of the manuscript and suggestions on writing of the paper.

\section{References}

[1] S. Bae, H. Jung and J. Ahn, 'Determinant formulas for class numbers in function fields', Math. Comp. 74 (2005), 935-965.

[2] S. Bae and P.-L. Kang, 'Class numbers of cyclotomic function fields', Acta Arith. 102 (2002), 251-259.

[3] D. R. Hayes, 'Explicit class field theory for rational function fields', Trans. Amer. Math. Soc. 189 (1974), 77-91. 
[4] M. Hirabayashi, 'A generalization of Maillet and Demyanenko determinants for the cyclotomic $\mathbb{Z}_{p}$-extension', Abh. Math. Sem. Univ. Hamburg 71 (2001), 15-27.

[5] H. Jung and $J$. Ahn, 'Demjanenko matrix and recursion formula for relative class number over function fields', J. Number Theory 98 (2003), 55-66.

[6] R. Kučera, 'Formulae for the relative class number of an imaginary abelian field in the form of a product of determinants', Acta Math. Inform. Univ. Ostraviensis 10 (2002), 79-83.

[7] M. B. Nathanson, Elementary methods in number theory, Graduate Texts in Mathematics 195 (Springer, New York, 2000).

[8] M. Rosen, 'A note on the relative class number in function fields', Proc. Amer. Math. Soc. 125 (1997), 1299-1303.

[9] - Number theory in function fields, Graduate Texts in Mathematics 210 (Springer, New York, 2002).

[10] H. Tsumura, 'A note on the Demjanenko matrices related to the cyclotomic $\mathbb{Z}_{p}$-extension', Proc. Japan Acad. Ser. A Math. Sci. 76 (2000), 99-103.

Department of Mathematics

KAIST

Daejon 305-701

Korea

e-mail: jaehyun@math.kaist.ac.kr young@math.kaist.ac.kr
Department of Mathematics Education Chungbuk National University Cheongju Chungbuk 361-763 Korea e-mail: hyjung@chungbuk.ac.kr 